\title{
Atualizações na doença de Parkinson através do tratamento com realidade virtual em 2018/2019
}

\author{
Updates in Parkinson's disease through virtual reality \\ treatment at 2018/2019
}

\section{Actualizaciones sobre la enfermedad de Parkinson a través del tratamiento de realidad virtual en 2018/2019}

\author{
Jessika Mehret Fiusa ${ }^{1}$, Jociléia Wilseque Zamboni ${ }^{2}$
}

1. Mestranda na Universidade Estadual de Londrina, Laboratório de engenharia neural e de reabilitação - LENeR. Londrina-PR, Brasil.

2.Pós-graduanda na Faculdade Inspirar, departamento de Fisioterapia Neurofuncional. Curitiba-PR, Brasil.

\begin{abstract}
Resumo
Introdução. A Doença de Parkinson (DP) consiste numa afecção neurológica progressiva, caracterizada pela degeneração do sistema nervoso central. Entre as técnicas de tratamento utilizadas na DP, a realidade virtual (RV) é utilizada com o intuito de realizar simulações interativas em tempo real. Objetivo. $O$ objetivo deste estudo foi verificar as técnicas mais utilizadas pelos pesquisadores atualmente na realidade virtual sobre a doença de Parkinson. Método: A revisão bibliográfica foi elaborada através da pesquisa nas bases Scielo, Bireme, Google scholar, PEdro e Cochrane, publicados no período de 2018 e 2019 com as palavraschave virtual reality and Parkinson's disease. Resultados. Foram utilizados 7 artigos, onde 0 maior interesse dos pesquisadores foi saber se a RV é melhor do que o tratamento convencional e qual equipamento tem maior eficácia. Os métodos de avaliação foram variados, incluindo testes de marcha, mobilidade, cognição e qualidade de vida. Utilizaram amostra em média de 28,7 indivíduos com tempo de tratamento médio de 12,6 sessões. A técnica de realidade virtual foi capaz de obter $85 \%$ de eficácia para os pacientes com DP. Conclusão. A realidade virtual é um conceituado método de tratamento para a doença de Parkinson. Sugerese a realização de pesquisas sobre a resposta da doença no momento em que o paciente será inserido ao ambiente real.
\end{abstract}

Unitermos. Doença de Parkinson; Realidade Virtual; Reabilitação; Fisioterapia

\begin{abstract}
Introduction. Parkinson's disease (PD) consists of a progressive neurological disorder characterized by degeneration of the central nervous system. Among the treatment techniques used in PD, virtual reality (VR) is used to perform interactive simulations in real time. Objective. The objective of this study was to verify the techniques most used by researchers currently in virtual reality on Parkinson's disease. Method. The bibliographic review was elaborated through the research in the databases Scielo, Bireme, Google scholar, PEdro and Cochrane, published in the period of 2018 and 2019 with keywords "reality virtual and Parkinson's disease". Results. We used 7 articles, where the greatest interest of the researchers was to know if VR is better than conventional treatment and which equipment is more effective. The evaluation methods were varied, including gait tests, mobility, cognition and quality of life. A mean sample of 28.7 individuals with mean treatment time of 12.6 sessions was used. The virtual reality technique was able to obtain $85 \%$ efficacy for PD patients. Conclusion. Virtual reality is a highly regarded method of treatment for Parkinson's disease. It is suggested to conduct research on the response of the disease now when the patient will be inserted into the real environment.
\end{abstract}

Keywords. Parkinson's disease; Virtual reality; Rehabilitation; Physiotherapy 


\section{Resumen}

Introducción. La enfermedad de Parkinson (EP) consiste en un trastorno neurológico progresivo caracterizado por la degeneración del sistema nervioso central. Entre las técnicas de tratamiento utilizadas en EP, la realidad virtual (RV) se utiliza para realizar simulaciones interactivas en tiempo real. Objetivo. El objetivo de este estudio fue verificar las técnicas más utilizadas por los investigadores actualmente en realidad virtual sobre la enfermedad de Parkinson. Método. La revisión bibliográfica fue elaborada a través de la investigación en las bases de datos Scielo, Bireme, Google scholar, PEdro y Cochrane, publicada en el período de 2018 y 2019 con las palabras clave virtuales realidad y la enfermedad de Parkinson. Resultados. Utilizamos 7 artículos, donde el mayor interés de los investigadores era saber si la RV es mejor que el tratamiento convencional y qué equipo es más eficaz. Los métodos de evaluación fueron variados, incluyendo pruebas de marcha, movilidad, cognición y calidad de vida. Se utilizó una muestra media de 28,7 individuos con un tiempo medio de tratamiento de 12,6 sesiones. La técnica de realidad virtual fue capaz de obtener un $85 \%$ de eficacia para pacientes con DP. Conclusión. La realidad virtual es un método de tratamiento muy apreciado para la enfermedad de Parkinson. Se sugiere realizar investigaciones sobre la respuesta de la enfermedad en el momento en que el paciente será insertado en el entorno real.

Palabras clave. Enfermedad de Parkinson; Realidad virtual; rehabilitación; fisioterapia

Trabalho realizado Universidade Estadual de Londrina. Londrina-PR, Brasil.

\section{INTRODUÇÃO}

A Doença de Parkinson (DP) consiste uma afecção neurológica progressiva, caracterizada pela degeneração do sistema nervoso central $^{1}$. Causa a morte neuronal da substância negra e reduz os níveis de dopamina². Existem mais de 50 sinais que caracterizam a doença, dentre estes o tremor em repouso, rigidez, bradicinesia, alterações de equilíbrio e dificuldade na marcha ${ }^{3}$.

Entre as técnicas de tratamento utilizadas na DP, temse a associação entre a administração medicamentosa para reposição de dopamina e a fisioterapia, a qual tem-se mostrado eficiente para a redução dos sintomas ${ }^{4}$. Dentre os recursos fisioterapêuticos, a realidade virtual (RV) é utilizada 
com o intuito de realizar simulações interativas, criadas com programas de computador.

A RV consiste em uma interface entre homem e máquina que possibilita a criação de um ambiente virtual ${ }^{5}$. Interage através de estímulos táteis, visuais, auditivos e sensoriais em tempo real. Permite a movimentação e interação em um ambiente tridimensional para treino de marcha, equilíbrio, coordenação motora, entre outros ${ }^{6}$.

A realidade virtual para o tratamento da doença de Parkinson, tem-se mostrado benéfica em diversos aspectos pois, envolve a possibilidade de incluir o indivíduo em ambientes que simulam objetos e eventos reais. Também proporciona o retorno do paciente ao convívio social 7 . Porém, dentro dessa técnica existem diversas formas de utilização, bem como diferentes tipos de equipamentos ${ }^{8}$.

Logo, o objetivo deste estudo foi verificar as técnicas mais utilizadas pelos pesquisadores atualmente na realidade virtual, por meio de revisão bibliográfica, para a reabilitação na doença de Parkinson.

\section{MÉTODO}

Esse estudo foi elaborado através da pesquisa nas bases de dados Scielo, Bireme, Google scholar, PEdro e Cochrane, publicados no período de 2018 e 2019. Utilizou-se as palavras-chave virtual reality and Parkinson's disease. 


\section{RESULTADOS e DISCUSSÃO}

Foram encontrados 19 artigos, dentre eles excluiu-se cinco repetidos e um por não estar disponível na forma de texto completo (Figura 1).

Figura 1. Número de artigos encontrados, repetidos, utilizados e excluídos através da pesquisa bibliográfica.

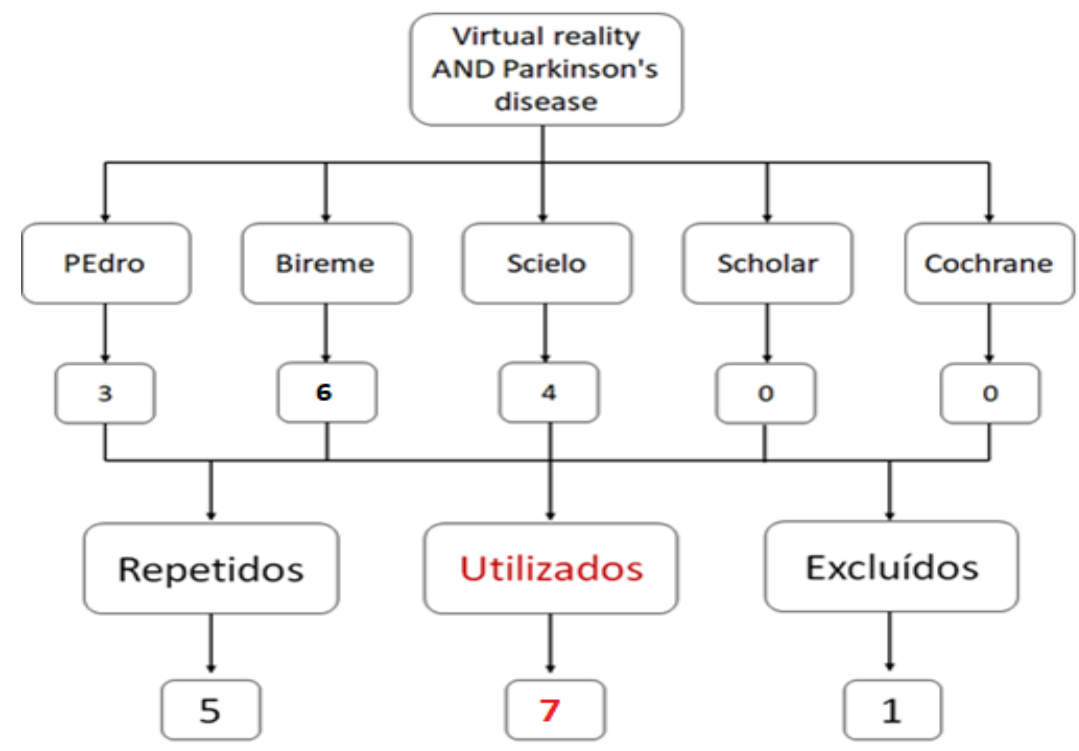

Dos 7 artigos utilizados (Figura 2), percebe-se que o maior interesse entre os pesquisadores foi saber se a RV é melhor do que o tratamento convencional e qual equipamento tem maior eficácia. Os métodos de avaliação foram variados, predominando testes de caminhada, mobilidade, aspectos cognitivos e questionários. Poucos são os artigos com análises quantitativas. Utilizaram amostra em média de 28,7 indivíduos com tempo de tratamento de 12,6 sessões. De acordo com os dados obtidos dos estudos do ano 
de 2018, observa-se que a técnica de realidade virtual foi capaz de obter $85 \%$ de eficácia.

Figura 2. Resultados referentes à revisão bibliográfica dos anos de 2018 e 2019 sobre realidade virtual e doença de Parkinson.

\begin{tabular}{|r|c|}
\hline Tipo de estudo & $48,8 \%$ controlado randomizado \\
\hline Ano & $100 \%$ em 2018 \\
\hline Amostra & Média de 28,7 pessoas \\
\hline Protocolo & $48,8 \%$ com Esteira x esteira e RV; $28,5 \%$ com Nintendo Wii \\
\hline Objetivo & $28,5 \%$ comparação entre técnicas; $28,5 \%$ cognição e qualidade de vida \\
\hline Avaliação & $57,1 \%$ testes de motricidade, $28,5 \%$ questionários \\
\hline Tempo de tratamento & 12,6 sessões em média \\
\hline Resultados & $85 \%$ de eficácia da RV \\
\hline
\end{tabular}

Alguns dos autores, realizaram estudos comparando o treino de marcha em esteira ergométrica versus esteira e realidade virtual ${ }^{9,10}$. Ambas as avaliações utilizaram técnicas atuais e inovadoras, sendo estimulação magnética transcraniana e espectroscopia no infravermelho próximo (NIRS). Seus resultados demonstraram maior efetividade no treino associado com RV, com a redução dos impulsos nervosos de repetição e maior controle de estabilidade postural durante a marcha.

Observaram-se que dois dos estudos encontrados tinham objetivos distintos, porém ambos avaliaram a partir de testes de caminhada, a fim de verificar velocidade da marcha, cadência, tempo de realização da atividade, cognição e motricidade ${ }^{8,11}$. Ambos também compararam duas técnicas de tratamento envolvendo a realidade virtual, 
onde o primeiro comparou esteira versus esteira com RV e o segundo comparou os equipamentos Nintendo $\mathrm{Wii}^{\mathrm{TM}}$ e $\mathrm{Xbox}$ Kinect $^{\mathrm{TM}}$ 8,11. O primeiro autor apresentou resultado semelhante ao de mais dois pesquisadores, com melhores efeitos durante o treino na esteira associada à $\mathrm{RV}^{9-11}$. O segundo autor, percebeu que Nintendo $\mathrm{Wii}^{\mathrm{TM}}$ foi capaz de promover melhores resultados para pacientes com Parkinson do que com Xbox Kinect ${ }^{T M}{ }^{8}$. Um terceiro pesquisador, realizou uma revisão bibliográfica sobre os efeitos do Nintendo $\mathrm{Wii}^{\mathrm{TM}}$ na reabilitação de indivíduos com DP e chegou à conclusão de que esse equipamento pode beneficiar os pacientes com melhora da marcha, motricidade e cognição ${ }^{12}$.

O estudo para verificar a eficácia dos exercícios de equilíbrio na DP associados com jogos de RV, avaliou seus participantes com alguns questionários e obteve melhora no seu padrão7. Outro estudo utilizou questionários para descrever seus resultados, com o objetivo de aumentar os níveis cognitivos e a qualidade de vida de seus participantes. Após duas semanas de intervenção com Xbox Kinect ${ }^{\mathrm{TM}}$ observou que houve uma leve melhora no aspecto cognição, porém o tratamento não acrescentou em qualidade de vida para os pacientes ${ }^{13}$.

\section{CONCLUSÃO}

Embora os estudos verificados nessa pesquisa tenham apresentado, em sua maioria resultados qualitativos, 
percebe-se que o uso da técnica de realidade virtual tem sido muito eficaz no auxílio do tratamento da doença de Parkinson.

No ano de 2018, os estudos na área basearam-se na comparação de técnicas, resultando, em todos os casos, na realidade virtual como melhor escolha para tratamento da doença de Parkinson utilizando o equipamento Nintendo Wii $^{\mathrm{TM}}$ para treino de marcha, mobilidade, cognição e qualidade de vida. O tempo de tratamento foi em média de 12,6 sessões com amostra de 28,7 indivíduos.

Sabe-se que a realidade virtual é um conceituado método de tratamento para a doença de Parkinson, verificado pelos pesquisadores citados nesse estudo. Entretanto, sugere-se a realização de pesquisas sobre a resposta da doença após o tratamento, momento em que o paciente será inserido ao ambiente real.

\section{REFERÊNCIAS}

1.Dixit S, Tedla JS. Effectiveness of robotics in improving upper extremity functions among people with neurological dysfunction: A Systematic Review. Int J Neurosci 2019;129:369-83. http://dx.doi.org/10.1080/00207454.2018

2. Postuma RB, Berg D, Stern M, Poewe W, Marek K, Litvan I, et al. MDS Clinical Diagnostic Criteria for Parkinson's Disease. Mov Dis 2015;30:1591-601. http://dx.doi.org/10.1002/mds.26424

3. Massano J. Doença de Parkinson. Acta Med Port 2011;24:827-34. file:///C:/Users/Ziggy/Downloads/1588-2237-1-PB.pdf

4. Capato TTC, Domingos JMM, Almeida LRS. Versão em Português da Diretriz Europeia de Fisioterapia para a Doença de Parkinson. São Paulo: OmniFarma, 2015.

https://www.parkinsonnet.nl/app/uploads/sites/3/2019/11/diretriz d $p$ brasil versao final publicada.pdf

5.Tori R, Kirner C, Siscouto R (eds). Fundamentos e tecnologia de Realidade Virtual e aumentada. Porto Alegre: Editora SBC. 2006. 
https://pcs.usp.br/interlab/wp-

content/uploads/sites/21/2018/01/Fundamentos e Tecnologia de R

ealidade Virtual e Aumentada-v22-11-06.pdf

6. Dockx K, Bekkers EM, Van den Bergh V, Ginis P, Rochester L, Jm H, et al. Virtual reality for rehabilitation in Parkinson 's disease. Cochrane Database Syst Rev 2016;12 CD010760.

http://dx.doi.org/10.1002/14651858.CD010760.pub2

7.Severiano MIR, Zeigelboim BS, Teive HAG, Santos GJB, Fonseca VR. Effect of virtual reality in Parkinson's disease: a prospective observational study. Arq Neuropsiquiatr 2018;78-84.

https://doi.org/10.1590/0004-282x20170195

8. Alves MLM, Mesquita BS, Morais WS, Leal JC, Satler CE, dos Santos Mendes Fa. Nintendo Wii ${ }^{\mathrm{TM}}$ Versus for Xbox Kinect ${ }^{\mathrm{TM}}$ Assisting People With Parkinson's Disease. Percept Mot Skills 2018;125:546-65. http://dx.doi.org/10.1177/0031512518769204

9.Pelosin E, Cerulli C, Ogliastro C, Lagravinese G, Mori L, Bonassi G, et al. A multimodal training modulates short-afferent inhibition and improves complex walking in a cohort of faller older adults with an increased prevalence of Parkinson's disease. J Gerontol A Biol Sci Med Sci 2020;75:722-8. http://dx.doi.org/10.1093/gerona/glz072

10.Maidan I, Nieuwhof F, Bernad-elazari H, Bloem BR, Giladi N, Hausdorff JM, et al. Evidence for Differential Effects of 2 Forms of Exercise on Prefrontal Plasticity During Walking in Parkinson's Disease. Neurorehabil Neural Repair 2018;32:200-8.

http://dx.doi.org/10.1177/1545968318763750

11.Ramos MTC, Costa LA, Silva LO, Costa TL, Melo RA. A Influência da Realidade Virtual no Equilíbrio e na Qualidade de Vida dos Pacientes com Doença de Parkinson. Rev Univ Vale do Rio Verde 2018;16:18. http://dx.doi.org/10.5892/ruvrd.v16i1.3529

12. Braz NFT, Dutra LR, Medeiros PES, Scianni AA, Faria CDCM. Eficácia do Nintendo Wii em desfechos funcionais e de saúde de indivíduos com doença de Parkinson: uma revisão sistemática. Fisioter Pesqui 2018;25:100-6. http://dx.doi.org/10.1590/18092950/17131825012018

13.Souza MFS, Bacha JMR, Silva KG, Feitas TB, Torriani-Pasin C, Pompeu JE. Effects of virtual rehabilitation on cognition and quality of life of patients with Parkinson's disease. Fisioter Mov 2018;31:1-9. https://doi.org/10.1590/1980-5918.031.ao12 